OPEN ACCESS

Edited by:

Paolo E. Porporato,

University of Turin, Italy

Reviewed by:

Valéry L. Payen,

Fonds National de la Recherche

Scientifique (FNRS), Belgium

Liwei Lang,

Augusta University, United States

*Correspondence:

Mehdi Damaghi

mehdi.damaghi@moffitt.org

Specialty section:

This article was submitted to

Cancer Metabolism

a section of the journal

Frontiers in Oncology

Received: 14 December 2019 Accepted: 03 March 2020

Published: 27 March 2020

Citation:

Ordway B, Swietach P, Gillies RJ and

Damaghi M (2020) Causes and Consequences of Variable Tumor Cell Metabolism on Heritable Modifications and Tumor Evolution. Front. Oncol. 10:373. doi: $10.3389 /$ fonc. 2020.00373

\section{Causes and Consequences of Variable Tumor Cell Metabolism on Heritable Modifications and Tumor Evolution}

\author{
Bryce Ordway ${ }^{1}$, Pawel Swietach ${ }^{2}$, Robert J. Gillies ${ }^{1}$ and Mehdi Damaghi ${ }^{1,3 *}$ \\ ' Department of Cancer Physiology, H Lee Moffitt Cancer Center and Research Institute, Tampa, FL, United States, \\ ${ }^{2}$ Department of Physiology, Anatomy and Genetics, University of Oxford, Oxford, United Kingdom, ${ }^{3}$ Department of \\ Oncologic Sciences, Morsani College of Medicine, University of South Florida, Tampa, FL, United States
}

When cancer research advanced into the post-genomic era, it was widely anticipated that the sought-after cure will be delivered promptly. Instead, it became apparent that an understanding of cancer genomics, alone, is unable to translate the wealth of information into successful cures. While gene sequencing has significantly improved our understanding of the natural history of cancer and identified candidates for therapeutic targets, it cannot predict the impact of the biological response to therapies. Hence, patients with a common mutational profile may respond differently to the same therapy, due in part to different microenvironments impacting on gene regulation. This complexity arises from a feedback circuit involving epigenetic modifications made to genes by the metabolic byproducts of cancer cells. New insights into epigenetic mechanisms, activated early in the process of carcinogenesis, have been able to describe phenotypes which cannot be inferred from mutational analyses per se. Epigenetic changes can propagate throughout a tumor via heritable modifications that have long-lasting consequences on ensuing phenotypes. Such heritable epigenetic changes can be evoked profoundly by cancer cell metabolites, which then exercise a broad remit of actions across all stages of carcinogenesis, culminating with a meaningful impact on the tumor's response to therapy. This review outlines some of the cross-talk between heritable epigenetic changes and tumor cell metabolism, and the consequences of such changes on tumor progression.

Keywords: tumor evolution, acidosis and oxidative stress, nutrient sensing and signaling, tumor micoenvironment, epigenetic regulation

\section{MOLECULAR MECHANISMS OF MICROENVIRONMENTAL SENSING}

Cancer evolution operates through selection, which requires a degree of phenotypic diversity to present a range of possible responses to microenvironmental selection forces, some of which confer selective advantage $(1,2)$. Tumors can be described as complete ecosystems, containing cancer cells, stromal cells, vasculature, extracellular matrix, and the chemical milieu consisting of variables such as $\mathrm{pH}$ and oxygen tension (3-5). During tumorigenesis-and similarly in response to therapy-the tumor ecosystem shows considerable plasticity because cancer cells shape their microenvironment, 
to which subsequent generations of them must adapt to thrive, and these adaptations, in turn, fine-tune the microenvironment (6). During the various stages of tumor progression, cells can be exposed to highly variable chemical stimulations, largely attributed to variable blood perfusion; for example, oxygen deprivation (hypoxia), nutrient deprivation, metabolic endproduct build-up, and increased acidity. Overall, these stimuli would be considered, by normal standards, to be survivable to most cells but exerts some cost on cells fitness, and it is therefore axiomatic that cancer cells must adapt to these conditions if they are to thrive. Although the stimuli are survivable, they still impose stress on the cells which changes their fitness, requiring acquisition of a novel homeostatic balance that costs more energy for cells and can be lethal for cells in competition with other cells.

Hypoxia is one of the main environmental factors a cancer cell must face in order to survive, thrive, and progress. Hypoxia imposes a metabolic stress on the cell, hindering its ability to carry out aerobic respiration. Therefore, the cell must be able to adapt to a hypoxic environment in order to survive. The cellular response to hypoxia is robust, and exerts most of its force via the transcription factors HIF- $1 \alpha$ and HIF- $2 \alpha$. Another HIF family protein, HIF-3 $\alpha$, functions to repress the responses directed by HIF- $1 \alpha$ and HIF- $2 \alpha$. All three of these proteins carry out their function via dimerization with constitutively expressed HIF $\beta$ proteins in the nucleus which allows them to directly modulate transcription of proteins involved in the hypoxia response $(7,8)$, or in the case of HIF- $3 \alpha$ repress the transcriptional response. HIF- $1 \alpha$ is a constitutively expressed protein, whose activity is regulated by the hydroxylation of conserved proline residues. In microenvironments of high oxygen tension $(>5 \%)$, the proline residues are hydroxylated, tagging the protein for degradation by E3 ubiquitin ligases (9). When oxygen concentrations are below the tolerable threshold for a given cell type, HIF- $1 \alpha$ is not degraded, and increases in concentration to allow HIF- $1 \alpha$ to induce transcription of its client genes (10).

Nutrient deprivation is another major stressor within the tumor microenvironment. When the cell experiences a critical reduction in a particular nutrient, it must swiftly adjust in order to maintain productivity in the terms of metabolism, proliferation, migration, or other processes essential to evolutionary success and survival. Two main nutrient sensing proteins implicated in cancer are AMPactivated protein kinase (AMPK) and the mammalian target of rapamycin (mTOR) $(11,12)$. These proteins are capable of sensing the current energy status of the cell and nutrient availability, respectively.

The metabolic pathway directed by AMPK is highly contextspecific; depending on the nutrient status of the cell. AMPK is responsible for the cellular response to glucose deprivation and acts as a metabolic switch from a highly glycolytic state to an oxidative state depending on the availability of glucose. This is particularly important in the context of cancer and the highly plastic nature of cancer metabolism. AMPK is activated by $5^{\prime}$ AMP, which indicates that the cell is not regenerating ATP at a fast-enough rate to meet demand. This induces the uptake of glucose and the induction of glycolysis to replenish the cellular ATP (11). The induction of glycolysis via-a-vis respiration is likely due to the promptness with which glycolytic activation can occur (13).

mTOR is present in the form of two different complexes, mTORC1 and mTORC2. These two complexes participate in associated, but distinct signaling pathways in nutrient sensing. mTORC1 becomes activated in response to various growth factors and amino acids that promote cell growth and proliferation. When inactive, mTORC1 represses growth and induces an autophagic response. mTORC2 is a sensor of glucose but also plays a role in amino acid signaling. mTORC2 is activated by acetyl-coenzyme A (Ac-CoA), which is produced in the cytoplasm via citrate lyase, when glycolytic flux is abundant. The result of mTORC2 activation is increased cell proliferation, in response to the increased glucose metabolism. mTORC2 has also been implicated in amino acid sensing by having the ability to suppress the function of the glutamine-cysteine transporter, system Xc transporter-related protein (12).

Aberrant perfusion in the tumor microenvironment allows a significant build-up of metabolites in the tumor interstitial fluid. The main metabolite that is commonly accumulated in the tumor interstitial fluid is lactic acid, which is associated with a decrease in $\mathrm{pH}$. A decrease in extracellular and intracellular $\mathrm{pH}$ can dramatically modulate the activities of enzymes, some of which are more sensitive than others, depending on how significant the change in $\mathrm{pH}$ is and the isoelectric point of the enzymes optimal activity (14). These alterations in enzyme activity are pleiotropic and leads to metabolic reprogramming. Lactate in the tumor microenvironment is a by-product of increased glucose fermentation, which occurs even in the presence of oxygen, known as aerobic glycolysis or the Warburg Effect. Once produced, lactate is shuttled out of the cell, stoichiometrically with a proton, by monocarboxylate transporters (MCTs) 1-4. Sensing of extracellular $\mathrm{pH}$ is accomplished through a variety of plasma membrane associated proteins including two major classes of acid-sensing receptors: (i) G-protein coupled receptors (GPCR) such as Ovarian cancer G protein-coupled receptor 1 (OGR1), G-protein coupled receptor 4 (GPR4), T-cell death-associated gene 8 (TDAG8), and ii) Acid-sensitive ion channels (ASICs) which include 7 proteins from 4 genes: $1 \mathrm{a} / \mathrm{b}, 2 \mathrm{a} / \mathrm{b}, 3,4,5$, and $\mathrm{Ca}^{2+}$ channel that includes transient receptor protein channel vanilloid subfamily 1 and 2 (TRPV1 and TRPV2) (15). Lactate can also be sensed and regulate cellular functions by activating the $\mathrm{G}$ protein-coupled receptors $\mathrm{HCA}_{1} / \mathrm{GPR} 81, \mathrm{HCA}_{2} / \mathrm{GPR} 109 \mathrm{~A}$, and $\mathrm{HCA}_{3} / \mathrm{GPR} 109 \mathrm{~B}$. These hydroxy-carboxylic acids (HCA) receptors control physiological homeostasis under changing metabolic and dietary conditions (16).

Cancer cells commonly overexpress many of the aforementioned acid sensors, and this can be correlated to tumor progression and poor outcome (17, 18). Therefore, investigating these sensors as a factor in malignancy may identify relevant prognostic biomarkers or may reveal new therapeutic vulnerabilities. The sensors can be connected to pathways to activate transcription factors or overexpress other genes and proteins (Figure 1). However, considering the ever-changing state of the microenvironment, we propose that epigenetic regulation may be a more effective factor in stabilization of emerging phenotypes in cancer cells. Adaptation 


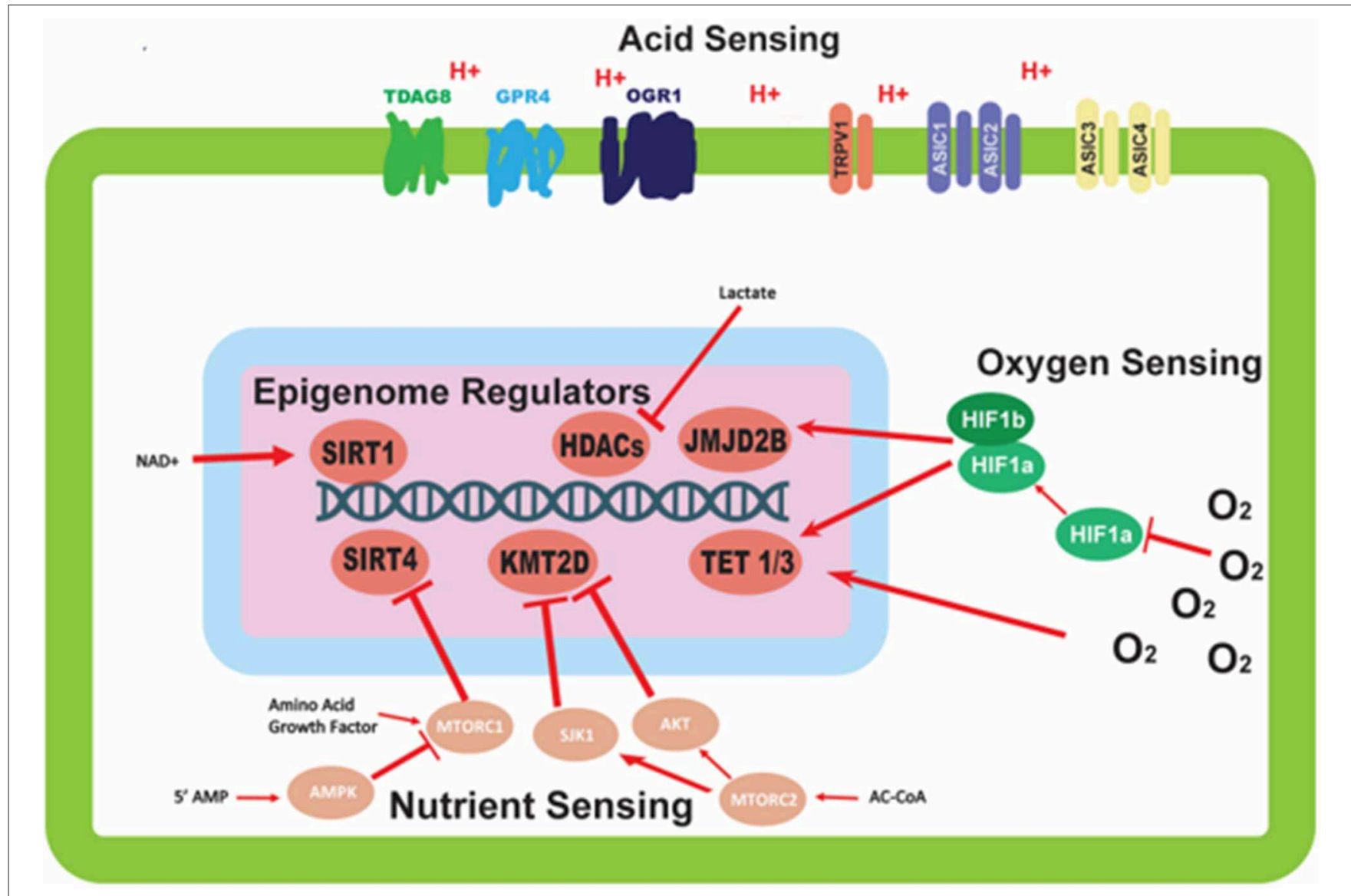

FIGURE 1 | Mechanisms of environmental sensing and their effects on epigenetic modifiers. This figure depicts the various avenues by which cells are able to detect perturbations in the environment, and the downstream effects of this sensing on epigenetic modifiers. Oxygen sensing mechanisms trigger dimerization of HIF-1 $\alpha$ and HIF-1 $\beta$ proteins to affect epigenetic modifiers; oxygen also direct modulates activity of epigenetic modifiers. Nutrient sensing can initiate a signaling cascade, largely mediated by the mTORC complexes and AMPK protein, with the ability to alter the activity of epigenetic modifying proteins. Acid sensing proteins located on the outer membrane of the cell are able to sense the extracellular $\mathrm{pH}$, but have no clear mechanism for altering the activity of epigenetic modifiers.

to an acid-microenvironment has been shown to alter cell state by pushing cells into a partial EMT phenotype (19); this may be a manifestiation of these acid sensors inducing a stable epigenetic change.

\section{HERITABLE EPIGENETIC MODIFICATIONS ACQUIRED THROUGH MICROENVIRONMENTAL SENSING}

One mechanism enabling cancer cells to adapt is through changes to gene expression via epigenetic regulation. Some modalities of epigenetic regulation are transient (e.g., histone acetylation) and are imposed to help cancer cells survive acute disruptions in their microenvironmental homeostasis. In contrast, other epigenetic mechanisms are more persistent (e.g., DNA methylation) and have the ability to be passed down through generations to endow further generations with memory on how to survive in the tumoral microenvironment.

The term epigenetics was first created by $\mathrm{CH}$ Waddington who described it as "the causal interactions between genes and their products, which bring the phenotype into being." While a commonly agreed upon definition is hard to find today, the term epigenetics in the modern era is commonly described as a permanent change in the way genes are expressed. Types of epigenetic regulation include histone modifications, DNA Methylation, and non-coding RNA (20), which can all impact one another to create a complex regulatory dynamic. A major question is: "How do the external factors of the tumoral microenvironment play into altering this complex dynamic"?

It is commonly known that the epigenetic signatures of cancer cells are different compared to their untransformed counterparts (21-25). Many of these epigenetic alterations exert their function by altering the metabolism of cancer cells (26), and are acquired by signaling cascades initiated by sensing of the extracellular environment. Some of the signaling cascades that can lead to changes of epigenetic signatures were implied in the previous section and the specific alterations they are involved in will be discussed herein.

In many solid tumors, intra-ductal hyperplasia leads to significant alterations in the physical microenvironment, especially in peri-luminal cells that are far (>160 microns) 
from their blood supplies. Importantly, the diffusion distance of oxygen in tissues is 100-200 microns (27), meaning that the periluminal cells of DCIS can be profoundly hypoxic. The depth and duration of hypoxia is dependent on the blood flow of the surrounding stroma. Hypoxia eventually selects for metabolic reprogramming, leading to acidosis, as well as exacerbating nutrient deprivation. Over many years in this environment, these forces (hypoxia, acidity, nutrient deprivation) select for cells with more highly adaptable, aggressive, de-differentiated phenotypes $(6,28)$. The resulting acidosis leads to genome instability, which could increase the rate of cancer evolution (29). The source of cytoplasmic (and nuclear) acidosis is lactate accumulation as a byproduct of glycolysis. Lactate has been shown to have a variety of effects on the epigenetic mechanisms of the cell, some of which are confounding. Direct inhibition of histone deacetylases (HDACs) by lactate has been shown in separate studies $(30,31)$; while others have reported multiple times on the increase in activity of the Sirtuin family of histone deacetylases upon exposure of cells to a chronically acidic extracellular environment $(32,33)$. These examples represent combined sensing and epigenetic effector mechanisms that act directly on altering the epigenetic status of the cell.

The oxygen sensing protein HIF- $1 \alpha$ can regulate epigenetics in a variety of ways. Upon activation, HIF- $1 \alpha$ leads to downstream signaling cascades important for the survival of cells in low oxygen environments. The ultimate effect of some of these signaling cascades is the epigenetic alteration of gene regulation (Figure 1). Two epigenetic mechanisms influenced by HIF- $1 \alpha$ are histone methylation and DNA methylation (Figure 1). Unlike other epigenetic mechanisms, histone methylation can act in both activating and repressing fashions depending on the specific location of the covalent modification. The histone demethylase JMJD2B is activated by HIF- $1 \alpha$ (Figure 1), and is specifically targeted to $\mathrm{H} 3 \mathrm{~K} 9 \mathrm{me} 2 / 3$ to demethylate the mark to a monomethylated state $(34,35)$. The expression of ten-eleven translocation proteins $1 / 3$ (TET1/3) is also upregulated upon stabilization of HIF-1 $\alpha$. TET1/3 are 5-methylcytosine $(5 \mathrm{mC})$ oxidases, which convert $5 \mathrm{mC}$ into either 5-hydroxymethylcytosine (5hmC), 5-formylcytosine (5fC), or 5-carboxylcytosine $(5 \mathrm{caC})$ via sequential reactions (36). The result of these reactions is the deactivation of the methylation mark and the subsequent reactivation of the sequence being repressed by the methylation. This has been validated in neuroblastoma where it was shown that HIF- $1 \alpha$ can induce HIF- $1 \alpha /$ hypoxia specific DNA methylation signatures (37). The fact that HIF- $1 \alpha$ activates DNA methylation supports our hypothesis of inheritable epigenetic changes to next generation that can be tracked in cancer cells. Contrary to the upregulation of TET by hypoxia-induced transcriptional programs, TET proteins have been shown to have their activity reduced directly by low oxygen availability in a tumor setting. TET activity is lost in vitro when exposed to hypoxic conditions, possibly via hypermethylation of tumor suppressor promoters in hypoxic regions of tumor samples (38). The opposing forces of the transcriptional and functional regulation of TET proteins may demonstrate a physiological feedback system for regulating the epigenetic response to oxygen deprivation in order to attenuate the response (Figure 1).

The sensing of nutrients by a cell is vitally important to its survival and can have long term effects on the downstream lineage of that cell via epigenetic modifications. Having this feed forward system of epigenetic regulation directed by nutrient signaling allows for increased fitness for subsequent generations. As mentioned previously, cellular nutrient sensing is mainly achieved through 3 essential proteins and protein complexes: AMPK, mTORC1, and mTORC2 (Figure 1). AMPK is activated in response to cellular metabolic stress, and modulates gene transcription epigenetically in order to respond to this stress. Unlike the mTORC1/2 complexes, AMPK is able to modulate transcription directly by phosphorylation of $\mathrm{Ser}^{36}$ on histone H2B (39). This phosphorylation mark directly promotes the transcription of response genes needed to handle metabolic stress. Other papers report the direct phosphorylation of $\mathrm{Ser}^{36}$ on $\mathrm{H} 2 \mathrm{~B}$ by S6K1 (40), which is also a player in the LKB1AMPK-mTORC1 signaling axis, with S6K1 being phosphorylated by mTORC1. mTORC1 is another player in the epigenetic response to nutrient sensing. As mentioned previously, mTORC1 is capable of sensing various growth factors and amino acids. A downstream target of mTORC1 nutrient sensing is SIRT4, which is repressed in response to mTORC1 activation (41). SIRT4 is a lysine deacylase (Figure 2) (42), that has the ability to inhibit glutamine metabolism by inhibiting glutamate dehydrogenase $(\mathrm{GDH})$. This inhibition of SIRT4 is achieved at the transcriptional level by mTORC1 stabilizing the CREB2$\beta \operatorname{TrCp}$ complex, preventing CREB2 from activating transcription of SIRT4 (41). mTORC2 exerts its epigenetic function by activation of the AKT and SGK1 proteins. The effect of these proteins on epigenetic regulation is the inhibition of KMT2D, which is a histone methyltransferase specifically targeting $\mathrm{H} 3 \mathrm{~K} 4$ (Figure 2) (43). Inhibition of KMT2D has been shown to have anti-tumor effects in some cancers by not allowing the FOXA1PBX-ER complex to access the DNA for transcription (44).

While the aforementioned mechanisms involve the sensing of nutrients to transduce downstream epigenetic changes, alterations in acetate level can directly influence the epigenetic status of the cell. Free Acetyl-CoA in the cell nucleus is produced by Acetyl-CoA synthetase (ACSS2), which catalyzes the conversion from Acetate, and by ATP-citrate lyase (ACLY) which catalyzes the conversion from citrate. The levels of free AcetylCoA directly influence the global acetylation status of histones (45), and henceforth have the ability to regulate epigenetics without the direct manipulation of an enzyme intermediate. While there is no direct sensing mechanism, this level of regulation could be seen as a sensor of the glycolytic state of the cell considering it has been shown that decreasing the amount of glucose available to a cell reduces the Acetyl-CoA abundance and lowers global histone acetylation (46).

A recently described mechanism of both environmental sensing and epigenetic modification is that of histone lactylation. In 2019, Zhang et al. described for the first time the modification of histones by lactate (47). As is commonly known, lactate is built-up as a byproduct of glycolysis. This epigenetic modification may provide a direct mechanism for the regulation 


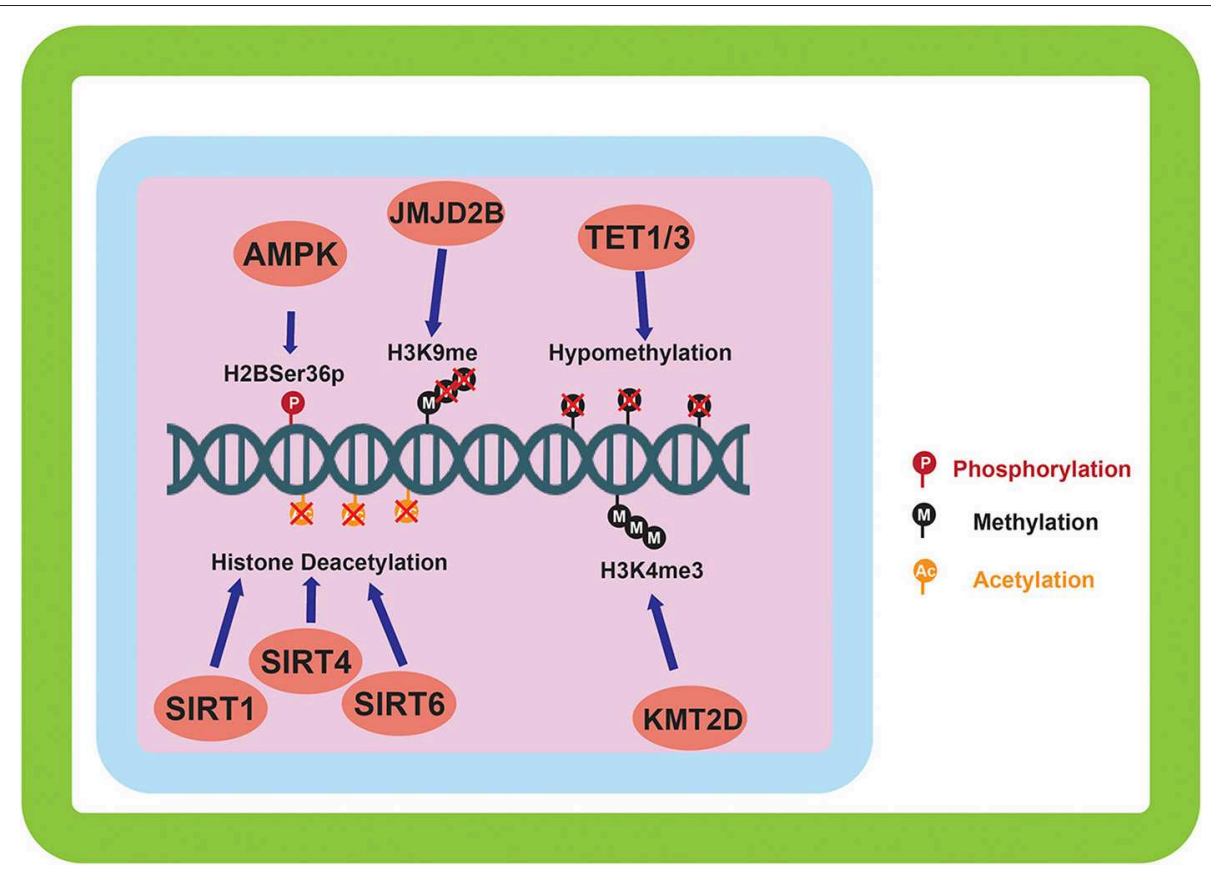

FIGURE 2 | Epigenetic alterations mediated by environmentally induced epigenetic modifiers. The epigenetic modifiers altered by the sensing of the cellular environment go on to carry out a variety of modifications to the epigenome. DNA methylation, and histone methylation and acetylation are the main downstream targets of the environmental sensing. Phosphorylation of histones by AMPK is also carried out in response to environmental sensing.

of gene expression in response to fermentative glycolytic activity of the cell.

\section{EFFECT OF HERITABLE EPIGENETIC MODIFICATIONS ON TUMOR METABOLISM}

Many epigenetic alterations that are acquired throughout tumor progression alter the metabolism of the tumor's cellular population. While the previous section covered specific epigenetic alterations that occur in response to the metabolic microenvironment, this section will describe the role of epigenetic modifications in altering cancer cell metabolism.

Lactate itself has the ability to alter the activity of epigenetic modifier proteins. The inhibition of HDAC's by lactate demonstrated in previous studies $(30,31)$ has yet to be phenotypically implicated in the alteration of metabolic processes; yet it is likely this lactate-mediated mechanism will play a role in altering metabolism. The activation of SIRT1 by extracellular acidosis, which is a consequence of acid-inhibition of glycolysis and the subsequent build-up of $\mathrm{NAD}^{+}$, has been shown to alter cellular metabolism through histone deacetylation leading to increased transcription of HIF- $2 \alpha$. This SIRT1/HIF$2 \alpha$ axis promotes the oxidative metabolism of glutamine, and suppresses the effects of HIF-1 $\alpha$, inhibiting hypoxia mediated induction of glycolysis (32). Corbet et al. in a later study showed that SIRT1 as well as SIRT6 are essential for histone deacetylation and the induction of fatty acid metabolism when cells are chronically exposed to an acidic extracellular environment (33). From a cell survival standpoint, this switch to other methods of energy metabolism when lactate has accumulated is intuitive, and has relevancy in the context of cancer progression that is discussed later.

In response to hypoxia, HIF-1 $\alpha$ activation leads to the induction of JMJD2B activity. JMJD2B has been shown to be upregulated in ER-positive breast cancer (48) and bladder cancer (49), and its upregulation has been directly linked to induction by HIF-1 $\alpha$ in colorectal cancer (50) and gastric cancer (51). This activation of JMJD2B directly drives the demethylation of $\mathrm{H} 3 \mathrm{~K} 9 \mathrm{me} 2 / 3$ to its monomethylated state (Figure 2). JMJD2B has been shown to play a role in altering the expression of many cancer associated genes including cyclin-dependent kinase 6 (CDK6) (49), and carbonic anhydrase 9 (CA9) (50), which can directly affect the transmembrane $\mathrm{pH}$ gradient. Also induced by hypoxia and HIF- $1 \alpha$ activation are the expression of TET proteins 1 and 3 (36). As mentioned previously, TET proteins are $5 \mathrm{mC}$ oxidases that allow for the expression of genes repressed by DNA methylation. Induction of TET in neuroblastoma has been shown to increase transcription of hypoxia response gene (52), and TET1 has been shown to be overexpressed in triple negative breast cancer (TNBC) where it is associated with hypomethylation (Figure 2) (53). Hypomethylation increases the expression of associated genes such as Hexokinase II (HK2) in liver cancer (54) and glioblastoma multiforme (55). Hexokinase II catalyzes the conversion of glucose to glucose-6-phosphate, an essential step in glycolysis. While CA9 and HK2 are both direct transcriptional targets of the HIF- $1 \alpha$ mediated hypoxic response, 
TABLE 1 | Represenation of the types of epigenetic modifications that can be induced by environmental sensing, and the specific modifications made.

\begin{tabular}{|c|c|c|c|c|c|}
\hline $\begin{array}{l}\text { Types of epigenetic } \\
\text { modifications }\end{array}$ & Half-Life (h) & Readers & Writers & Erasers & References \\
\hline DNA Methylation & Indefinite & $\begin{array}{l}\text { MeCP2, MBD1/2/3/4, UHRF1/2, } \\
\text { Kaiso, ZBTB4/38 }\end{array}$ & Dnmt1/3a/3b & TET1-3 & $(70-73)$ \\
\hline Histone Acetylation & $0.88-1.45$ & $\begin{array}{l}\text { Bromodomain containing } \\
\text { enzymes }\end{array}$ & HATs & HDACs, SIRT1-7 & $(74-76)$ \\
\hline Histone Methylation & 5 & $\begin{array}{l}\text { Proteins containing PHD, } \\
\text { chromo, tudor, PWWP, WD40, } \\
\text { BAH, ADD, ankyrin repeat, MBT, } \\
\text { and zn-CW domains. }\end{array}$ & $\begin{array}{l}\text { Histone } \\
\text { Methyltransferases }\end{array}$ & $\begin{array}{l}\text { KDM1 Family proteins, and } \\
\text { JMJC Domain-Containing } \\
\text { Demthylases }\end{array}$ & $(77-81)$ \\
\hline H3K9me3 & 221 & PRC Complex & SUV39H1, SETDB1/2 & JMJD2/KDM4, LSD1 & $(82,83)$ \\
\hline H3K27me3 & 158 & BAHD1 & $\mathrm{EZH} 2$ & JMJD3 & $(40,82,84,85)$ \\
\hline H3K9me2 & 28.5 & PRC Complex & $\begin{array}{l}\text { G9a/GLP, PRDM } \\
\text { Family }\end{array}$ & JMJD2/KDM4 & $(82,83)$ \\
\hline Histone Lactylation & Unknown & Unknown & Lactate Accumulation & Unknown & $(47)$ \\
\hline \multicolumn{6}{|c|}{ Histone Phosphorylation } \\
\hline H2BSer ${ }^{36}$ & Unknown & Unknown & AMPK, S6K1 & Unknown & $(39,40)$ \\
\hline
\end{tabular}

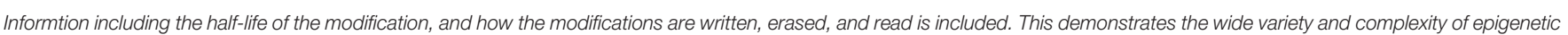
modifications that can be made in response to sensing, and the time-scales at which their effects are applicable.

this epigenetic regulation is important because it imposes the upregulation of these proteins over longer time scales (Table 1) and allows for the maintenance of the metabolic phenotype independently of oxygen status.

Epigenetic marks induced by nutrient sensing proteins and complexes have the ability to greatly alter cellular metabolism, making a useful feed-forward mechanism for acclimation and adaptation to the current metabolic microenvironment. The phosphorylation of $\mathrm{Ser}^{36}$ on Histone $2 \mathrm{~B}$ is a significant epigenetic mark made by two proteins involved in nutrient sensing: AMPK and S6K1 $(39,40)$. It has been shown that phosphorylation of $\mathrm{Ser}^{36}$ on Histone $2 \mathrm{~B}$ is significantly increased upon treatment of cells with 2-Deoxy Glucose (39), which mimics a glucose deprived environment. The resulting effect on the cellular transcription from phosphorylation of $\mathrm{Ser}^{36}$ on Histone $2 \mathrm{~B}$ by AMPK is the recruitment of EZH2 (40). EZH2 is a histone methyltransferase that trimethylates Lysine 27 on histone 3 when recruited. It has been shown in Drosophila that trimethylation of Lysine 27 on histone 3 reduces the glycolytic tendencies of the cell (56). Considering the presence of this mark in glucosedeprived cellular states, it would intuitively make sense that the presence of this mark would decrease the glycolytic capacity of the cell.

The suppression of SIRT4 by mTORC1 has profound effects on the metabolism of cancer cells, specifically inhibiting glutamine metabolism through inhibition of GDH. In colorectal cancer, decreased SIRT4 expression has been correlated with progression and increased invasive potential of cancer cells (57), and in both colorectal and gastric cancers lower SIRT4 expression is associated with poor prognosis $(57,58)$. All in all, this leads to the conclusion that when in the presence of sufficient amino acids and growth factors, the activation of mTORC1 will lead to the inhibition of SIRT4 and the subsequent reactivation of glutamine metabolism which can promote tumor growth (Figure 2).
mTORC2 has the ability to modulate activity of KMT2D. KMT2D is inhibited downstream during mTORC2 activation, which in response, inhibits the access of the FOXA1-PBX1-ER complex from binding the DNA. Prevention of this complex from binding the DNA has been shown to reduce the expression of key proteins including: GREB1, SERPINA1, cFOS, and MYC (59). Of these proteins, MYC has been shown to have the most substantial effects on reprogramming cancer metabolism in a type-specific manner. A comprehensive list of metabolic alterations in specific cancer types driven by MYC has recently been reviewed (60). The effect MYC has on glycolysis is highly variable depending on the cancer type, with a MYC-associated increase in non-small cell lung cancer and hepatocellular carcinoma, and a MYC associated decrease in renal cell carcinoma and prostatic intraepithelial neoplasia. MYC's effect on glutaminolysis was cohesive in the various cancer types, with an associated increase demonstrated in hepatocellular carcinoma, pancreatic ductal adenocarcinoma, and renal cell carcinoma.

In addition to the previously mentioned alterations in gene expression caused by stimulus-induced epigenetic modifications, epigenetic upregulation of MCT4 via hypomethylation of the SLC16A3 promoter has been shown in renal cancers (61). Although no specific mechanism can be tied to this alteration, this increase in MCT4 expression will have profound consequences on the long-term progression and evolution of the tumor.

\section{CONSEQUENCES OF EPIGENETICALLY ALTERED METABOLISM ON TUMOR PROGRESSION}

As described previously, a resulting consequence of oxygen deprivation and stabilization of HIF- $1 \alpha$ is the induction of TET1/3 and hypomethylation of the genome. Hypomethylation 


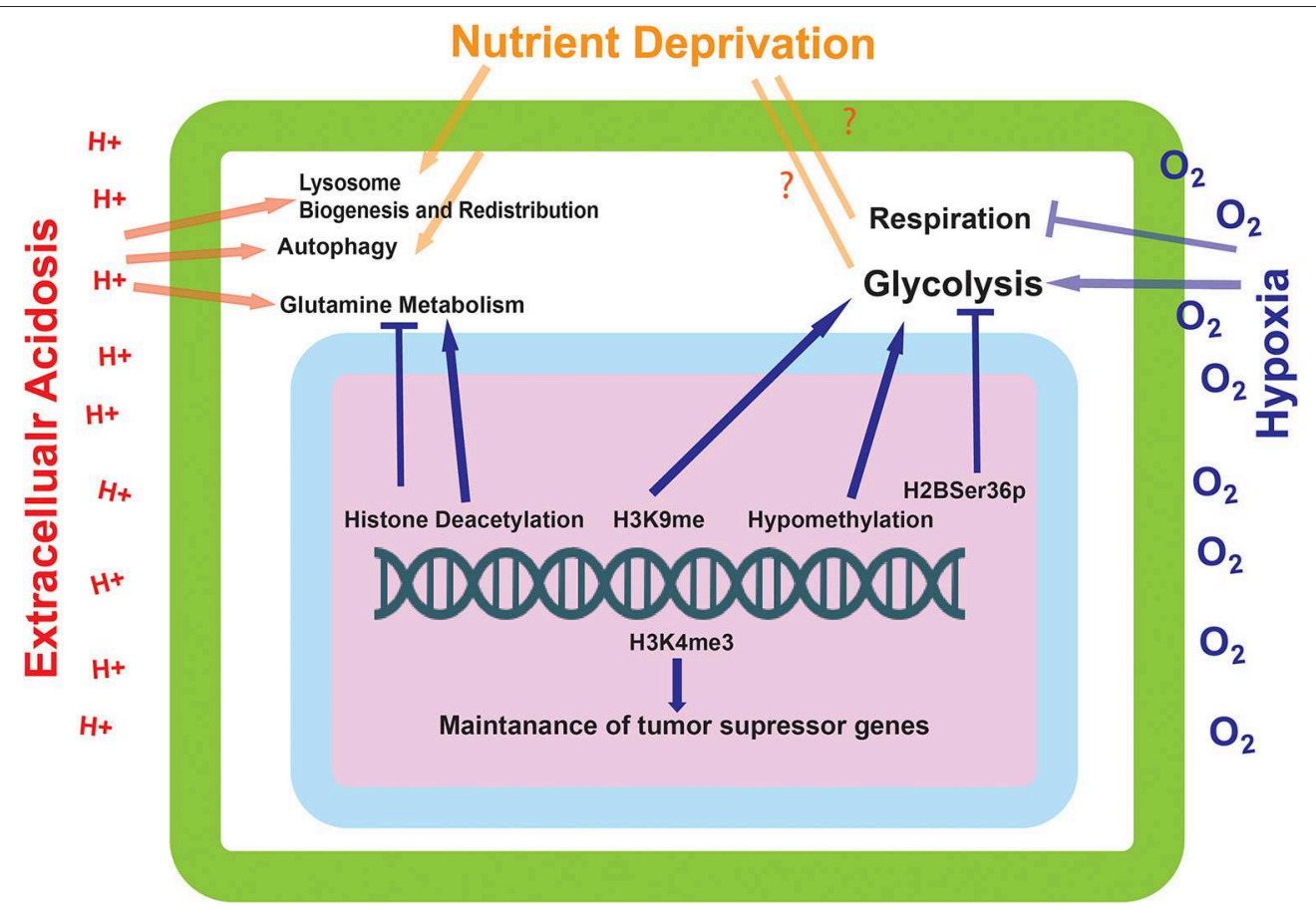

FIGURE 3 | The effect of environmentally induced epigenetic modifications on tumor cell expression and metabolism. The epigenetic modifications made by the proteins influenced by the environmental sensing mechanisms go on to alter the metabolic state of the cell. These modifications can alter the cells ability to metabolize glutamine and carry out glycolysis, as well as influence the transcriptional status of tumor suppressor genes.

has been shown to upregulate the expression of Hexokinase $2(54,55)$, which is associated with an increase in glycolysis (Figure 3). Increased glycolysis will increase acidosis in the tumor microenvironment that can induce extracellular matrix remodeling (15). Thus, increased glycolysis and its sequelae are barriers that cancer cells must overcome in order to meet the energy demands of rapid proliferation and to survive and thrive in a more hostile environment. Altered glycolysis can also lead to Warburg phenotype leading to even more acidic microenvironment and more altered genome and epigenome alteration $(28,62)$.

Despite the differing sensing mechanisms of the mTORC1/mTORC2 complexes and SIRT1, all mechanisms converge on a single metabolic alteration caused by epigenetic modification: the increase of glutamine metabolism. This may pose the opportunity to target glutamine metabolism as a cancer therapeutic, an idea that has drawn enough attention to warrant various reviews on that subject alone $(63,64)$. Although this may seem like a rational therapeutic target, cautionary narratives have been proposed as to the possible outcome of creating a resistant cellular population with a heightened metabolic capacity (65). While glutamine metabolism is non-essential in a normally proliferating cell, under periods of rapid proliferation, like tumor growth, glutaminolysis is an essential process (66). This phenotype is selected for due to the high demand for metabolic building blocks produced from the TCA cycle. In the TCA cycle alpha-ketoglutarate can be carboxylated to citrate, which, if in abundance, is translocated to the cytoplasm where it is used for fatty acid synthesis. The end product of glutaminolysis is alpha-ketoglutarate, which is shunted into the TCA cycle to accelerate the process (67). Supporting the TCA cycle with the necessary building blocks will give more chance to glucose to be turned into lactate in glycolysis and augment a Warburg phenotype. Glutamine-fed TCA cycle can also give more freedom to cancer cells to use glycolysis for their fluctuating ATP demand as a quick local source of energy for cancer cells (13).

\section{DISCUSSION}

Genomic data describing tumors and cancer cell populations is valuable information for studying and classifying a cancers phenotypic characteristics. Although this information is valuable, it does not tell the entire story when it comes to cancer initiation and progression. Recent studies have demonstrated the presence of single and multiple driver mutations in significant proportions of cells comprising healthy tissue $(68,69)$. This poses the question as to how cancer arises, as the once thought sufficient accumulation of driver mutations has been discounted. While it is no doubt these mutations are necessary and play a significant role in cancer progression, it is clear there is more to the story.

Metabolic cross-talk and feedback is essential for the survival of any cell in a highly variable environment. There are many ways cells can sense perturbations in their microenvironment, including nutrient, and energy demands. These sensing mechanisms can transduce signals that lead to the alteration of 
the cell's epigenome. The epigenetic alterations driven by the alterations to the cellular environment and metabolic state can go on to influence the metabolism of the cell in a feed-forward mechanism. These changes made can have long lasting and heritable effects and go on to influence the progression of the tumor.

Nutrient sensing by cells is essential for the survival and acclimation to an unstable environment (Figure 3). Permanent changes induced by a given environment may be beneficial to a cells lineage, in that they are pre-programmed to deal with the environments endured by their predecessors. In cancer, cells are dynamically exposed to a variety of environmental conditions that would be extremely difficult to survive without epigenetic acclimation handed down from predecessors. Founding cancer populations may have a difficult time surviving the variety of harsh environmental factors, but genetic reprogramming facilitated by epigenetic change would allow the daughter populations to have an increased fitness.

The hyper-glycolytic state of cancer cells is a hallmark of their progression and aggressive state (28). At present, a conclusive mechanism as to the induction of this glycolytic state has yet to be achieved. Described in this paper are various mechanisms in which a cell is able to sense its' current environmental and energetic state of being, some of which can lead to long-lasting changes in the metabolic programming of a cell. Many of these semi-permanent changes converge on the regulation of cellular

\section{REFERENCES}

1. Lorusso G, Ruegg C. The tumor microenvironment and its contribution to tumor evolution toward metastasis. Histochem Cell Biol. (2008) 130:10913. doi: 10.1007/s00418-008-0530-8

2. Yuan Y. Spatial heterogeneity in the tumor microenvironment. Cold Spring Harb Perspect Med. (2016) 6:a026583. doi: 10.1101/cshperspect.a026583

3. Gillies RJ, Gatenby RA. Hypoxia and adaptive landscapes in the evolution of carcinogenesis. Cancer Metastasis Rev. (2007) 26:311-7. doi: 10.1007/s10555-007-9065-Z

4. Gillies RJ, Gatenby RA. Adaptive landscapes and emergent phenotypes: why do cancers have high glycolysis? J Bioenerg Biomembr. (2007) 39:2517. doi: 10.1007/s10863-007-9085-y

5. Gatenby RA, Gillies RJ, Brown JS. Evolutionary dynamics of cancer prevention. Nature Rev Cancer. (2010) 10:526-7. doi: 10.1038/nrc2892

6. Gatenby RA, Gillies RJ. A microenvironmental model of carcinogenesis. Nature Rev Cancer. (2008) 8:56-61. doi: 10.1038/nrc 2255

7. Pouyssegur J, Dayan F, Mazure NM. Hypoxia signalling in cancer and approaches to enforce tumour regression. Nature. (2006) 441:43743. doi: $10.1038 /$ nature 04871

8. Semenza GL. Life with oxygen. Science. (2007) 318:624. doi: 10.1126/science.1147949

9. Bracken CP, Fedele AO, Linke S, Balrak W, Lisy K, Whitelaw ML, et al. Cellspecific regulation of hypoxia-inducible factor (HIF)-1alpha and HIF-2alpha stabilization and transactivation in a graded oxygen environment. J Biol Chem. (2006) 281:22575-85. doi: 10.1074/jbc.M600288200

10. Majmundar AJ, Wong WJ, Simon MC. Hypoxia-inducible factors and the response to hypoxic stress. Mol Cell. (2010) 40:294-309. doi: 10.1016/j.molcel.2010.09.022

11. Hardie DG. Molecular pathways: is AMPK a friend or a foe in cancer? Clin Cancer Res. (2015) 21:3836-40. doi: 10.1158/1078-0432.CCR-143300 energetics, in particular, glycolysis. It is therefore conceivable that the mechanism for the induction of a glycolytic state in cancer cells may not be a proposed "switch" or genetic mutation, but instead the accumulation of various epigenetic alterations that permanently reprogram the cellular population (Figure 3). It is possible that this reprogramming would occur early on in tumorigenesis, caused by a lack of perfusion in the core of the tumor which would cause many of the environmental perturbations that induce the epigenetic alterations described in this paper. What is certain, is that the spatial and temporal aspects of these epigenetic modifications would be vitally important for directing tumor progression. More studies need to be completed in order to elucidate how these epigenetic changes occur directly in relation to tumor growth in models and in the patient.

\section{AUTHOR CONTRIBUTIONS}

MD and RG developed the idea. All authors wrote the manuscript.

\section{FUNDING}

This work was supported by grants: U54CA193489, Cancer as a Complex Adaptive System, and P30CA076292, Moffitt Cancer Center Support Grant.
12. Harachi M, Masui K, Okamura Y, Tsukui R, Mischel PS, Shibata N. mTOR complexes as a nutrient sensor for driving cancer progression. Int J Mol Sci. (2018) 19: E3267. doi: 10.3390/ijms19103267

13. Epstein T, Xu L, Gillies RJ, Gatenby RA. Separation of metabolic supply and demand: aerobic glycolysis as a normal physiological response to fluctuating energetic demands in the membrane. Cancer Metab. (2014) 2:7. doi: 10.1186/2049-3002-2-7

14. Persi E, Duran-Frigola M, Damaghi M, Roush WR, Aloy P, Cleveland JL, et al. Systems analysis of intracellular $\mathrm{pH}$ vulnerabilities for cancer therapy. Nat Commun. (2018) 9:2997. doi: 10.1038/s41467-018-05261-x

15. Damaghi M, Wojtkowiak JW, Gillies RJ. pH sensing and regulation in cancer. Front Physiol. (2013) 4:370. doi: 10.3389/fphys.2013.00370

16. Offermanns S. Hydroxy-carboxylic acid receptor actions in metabolism. Trends Endocrinol Metab. (2017) 28:227-36. doi: 10.1016/j.tem.2016. 11.007

17. Wu Y, Gao B, Xiong QJ, Wang YC, Huang DK, Wu WN. Acidsensing ion channels contribute to the effect of extracellular acidosis on proliferation and migration of A549 cells. Tumour Biol. (2017) 39:1010428317705750. doi: 10.1177/1010428317705750

18. Zhou ZH, Song JW, Li W, Liu X, Cao L, Wan LM, et al. The acid-sensing ion channel, ASIC2, promotes invasion and metastasis of colorectal cancer under acidosis by activating the calcineurin/NFAT1 axis. J Exp Clin Cancer Res. (2017) 36:130. doi: 10.1186/s13046-017-0599-9

19. Sadeghi M, Ordway B, Rafiei I, Borad P, Fang B, Koomen JL, et al. Integrative analysis of breast cancer cells reveals an epithelial-mesenchymal transition role in adaptation to acidic microenvironment. Front Oncol. (2020) 10:304 doi: 10.3389/fonc.2020.00304

20. Handy DE, Castro R, Loscalzo J. Epigenetic modifications: basic mechanisms and role in cardiovascular disease. Circulation. (2011) 123:2145-56. doi: 10.1161/CIRCULATIONAHA.110.956839

21. Feinberg AP, Vogelstein B. Hypomethylation distinguishes genes of some human cancers from their normal counterparts. Nature. (1983) 301:8992. doi: $10.1038 / 301089 \mathrm{a} 0$ 
22. Feinberg AP, Vogelstein B. Hypomethylation of ras oncogenes in primary human cancers. Biochem Biophys Res Commun. (1983) 111:47-54. doi: 10.1016/S0006-291X(83)80115-6

23. Goelz SE, Vogelstein B, Hamilton SR, Feinberg AP. Hypomethylation of DNA from benign and malignant human colon neoplasms. Science. (1985) 228:187-90. doi: 10.1126/science.2579435

24. Cheah MS, Wallace CD, Hoffman RM. Hypomethylation of DNA in human cancer cells: a site-specific change in the c-myc oncogene. J Natl Cancer Inst. (1984) 73:1057-65. doi: 10.1093/jnci/73.5.1057

25. Cox R, Tuck MT. Alteration of methylation patterns in rat liver histones following administration of ethionine, a liver carcinogen. Cancer Res. (1981) 41:1253-6

26. Miranda-Goncalves V, Lameirinhas A, Henrique R, Jeronimo C. Corrigendum: metabolism and epigenetic interplay in cancer: regulation and putative therapeutic targets. Front Genet. (2019) 10:784. doi: 10.3389/fgene.2019.00784

27. Helmlinger G, Yuan F, Dellian M, Jain RK. Interstitial $\mathrm{pH}$ and $\mathrm{pO} 2$ gradients in solid tumors in vivo: high-resolution measurements reveal a lack of correlation. Nat Med. (1997) 3:177-82. doi: 10.1038/nm0297-177

28. Gatenby RA, Gillies RJ. Why do cancers have high aerobic glycolysis? Nat Rev Cancer. (2004) 4:891-9. doi: 10.1038/nrc1478

29. Tafreshi NK, Gillies RJ, Morse DL. Molecular imaging of breast cancer lymph node metastasis. Eur J Radiol. (2012) 81 (Suppl. 1):S160-1. doi: 10.1016/S0720-048X(12)70067-0

30. Latham T, Mackay L, Sproul D, Karim M, Culley J, Harrison DJ, et al. Lactate, a product of glycolytic metabolism, inhibits histone deacetylase activity and promotes changes in gene expression. Nucleic Acids Res. (2012) 40:4794803. doi: $10.1093 /$ nar/gks066

31. Wagner W, Ciszewski WM, Kania KD. L- and D-lactate enhance DNA repair and modulate the resistance of cervical carcinoma cells to anticancer drugs via histone deacetylase inhibition and hydroxycarboxylic acid receptor 1 activation. Cell Commun Signal. (2015) 13:36. doi: 10.1186/s12964-015-0114-x

32. Corbet C, Draoui N, Polet F, Pinto A, Drozak X, Riant O, et al. The SIRT1/HIF2 $\alpha$ axis drives reductive glutamine metabolism under chronic acidosis and alters tumor response to therapy. Cancer Res. (2014) 74:550719. doi: 10.1158/0008-5472.CAN-14-0705

33. Corbet C, Pinto A, Martherus R, Santiago de Jesus JP, Polet F, Feron O. Acidosis drives the reprogramming of fatty acid metabolism in cancer cells through changes in mitochondrial and histone acetylation. Cell Metab. (2016) 24:311-23. doi: 10.1016/j.cmet.2016.07.003

34. Fodor BD, Kubicek S, Yonezawa M, O’Sullivan RJ, Sengupta R, Perez-Burgos L, et al. Jmjd2b antagonizes H3K9 trimethylation at pericentric heterochromatin in mammalian cells. Genes Dev. (2006) 20:1557-62. doi: 10.1101/gad.388206

35. Whetstine JR, Nottke A, Lan F, Huarte M, Smolikov S, Chen Z, et al. Reversal of histone lysine trimethylation by the JMJD2 family of histone demethylases. Cell. (2006) 125:467-81. doi: 10.1016/j.cell.2006.03.028

36. Wu $\mathrm{H}$, Zhang $\mathrm{Y}$. Mechanisms and functions of Tet proteinmediated 5-methylcytosine oxidation. Genes Dev. (2011) 25:243652. doi: $10.1101 /$ gad.179184.111

37. Cimmino F, Avitabile M, Lasorsa VA, Montella A, Pezone L, Cantalupo S, et al. HIF-1 transcription activity: HIF1A driven response in normoxia and in hypoxia. BMC Med Genet. (2019) 20:37. doi: 10.1186/s12881-0190767-1

38. Thienpont B, Steinbacher J, Zhao H, D’Anna F, Kuchnio A, Ploumakis A, et al. Tumour hypoxia causes DNA hypermethylation by reducing TET activity. Nature. (2016) 537:63-8. doi: 10.1038/nature19081

39. Bungard D, Fuerth BJ, Zeng PY, Faubert B, Maas NL, Viollet B, et al. Signaling kinase AMPK activates stress-promoted transcription via histone $\mathrm{H} 2 \mathrm{~B}$ phosphorylation. Science. (2010) 329:1201-5. doi: 10.1126/science.1191241

40. Yi SA, Um SH, Lee J, Yoo JH, Bang SY, Park EK, et al. S6K1 phosphorylation of $\mathrm{H} 2 \mathrm{~B}$ mediates $\mathrm{EZH} 2$ trimethylation of $\mathrm{H} 3$ : a determinant of early adipogenesis. Mol Cell. (2016) 62:443-52. doi: 10.1016/j.molcel.2016. 03.011

41. Csibi A, Fendt SM, Li C, Poulogiannis G, Choo AY, Chapski DJ, et al. The mTORC1 pathway stimulates glutamine metabolism and cell proliferation by repressing SIRT4. Cell. (2013) 153:840-54. doi: 10.1016/j.cell.2013.04.023
42. Anderson KA, Huynh FK, Fisher-Wellman K, Stuart JD, Peterson BS, Douros JD, et al. SIRT4 is a lysine deacylase that controls leucine metabolism and insulin secretion. Cell Metab. (2017) 25:838-55 e15. doi: 10.1016/j.cmet.2017.03.003

43. Toska E, Castel P, Chhangawala S, Arruabarrena-Aristorena A, Chan C, Hristidis VC, et al. PI3K inhibition activates SGK1 via a feedback loop to promote chromatin-based regulation of ER-dependent gene expression. Cell Rep. (2019) 27:294-306.e5. doi: 10.1016/j.celrep.2019.02.111

44. Koren S, Bentires-Alj M. Tackling resistance to PI3K inhibition by targeting the epigenome. Cancer Cell. (2017) 31:616-8. doi: 10.1016/j.ccell.2017.04.010

45. Sidoli S, Trefely S, Garcia BA, Carrer A. Integrated analysis of AcetylCoA and histone modification via mass spectrometry to investigate metabolically driven acetylation. Methods Mol Biol. (2019) 1928:12547. doi: 10.1007/978-1-4939-9027-6_9

46. Lee JV, Carrer A, Shah S, Snyder NW, Wei S, Venneti S, et al. Aktdependent metabolic reprogramming regulates tumor cell histone acetylation. Cell Metab. (2014) 20:306-19. doi: 10.1016/j.cmet.2014.06.004

47. Zhang D, Tang Z, Huang H, Zhou G, Cui C, Weng Y, et al. Metabolic regulation of gene expression by histone lactylation. Nature. (2019) 574:57580. doi: 10.1038/s41586-019-1678-1

48. Kawazu M, Saso K, Tong KI, McQuire T, Goto K, Son DO, et al. Histone demethylase JMJD2B functions as a co-factor of estrogen receptor in breast cancer proliferation and mammary gland development. PLOS ONE. (2011) 6:e17830. doi: 10.1371/journal.pone.0017830

49. Toyokawa G, Cho HS, Iwai Y, Yoshimatsu M, Takawa M, Hayami S, et al. The histone demethylase JMJD2B plays an essential role in human carcinogenesis through positive regulation of cyclin-dependent kinase 6. Cancer Prev Res. (2011) 4:2051-61. doi: 10.1158/1940-6207.CAPR-11-0290

50. Fu L, Chen L, Yang J, Ye T, Chen Y, Fang J. HIF- $1 \alpha$-induced histone demethylase JMJD2B contributes to the malignant phenotype of colorectal cancer cells via an epigenetic mechanism. Carcinogenesis. (2012) 33:166473. doi: $10.1093 /$ carcin/bgs217

51. Kim JG, Yi JM, Park SJ, Kim JS, Son TG, Yang K, et al. Histone demethylase JMJD2B-mediated cell proliferation regulated by hypoxia and radiation in gastric cancer cell. Biochim Biophys Acta. (2012) 1819:12007. doi: 10.1016/j.bbagrm.2012.10.001

52. Mariani CJ, Vasanthakumar A, Madzo J, Yesilkanal A, Bhagat T, Yu Y, et al. TET1-mediated hydroxymethylation facilitates hypoxic gene induction in neuroblastoma. Cell Rep. (2014) 7:1343-52. doi: 10.1016/j.celrep.2014.04.040

53. Good CR, Panjarian S, Kelly AD, Madzo J, Patel B, Jelinek $\mathrm{J}$, et al. TET1-mediated hypomethylation activates oncogenic signaling in triple-negative breast cancer. Cancer Res. (2018) 78:4126-37. doi: 10.1158/0008-5472.CAN-17-2082

54. Goel A, Mathupala SP, Pedersen PL. Glucose metabolism in cancer. Evidence that demethylation events play a role in activating type II hexokinase gene expression. J Biol Chem. (2003) 278:15333-40. doi: 10.1074/jbc.M300608200

55. Wolf A, Agnihotri S, Munoz D, Guha A. Developmental profile and regulation of the glycolytic enzyme hexokinase 2 in normal brain and glioblastoma multiforme. Neurobiol Dis. (2011) 44:84-91. doi: 10.1016/j.nbd.2011.06.007

56. Ma Z, Wang H, Cai Y, Wang H, Niu K, Wu X, et al. Epigenetic drift of H3K27me3 in aging links glycolysis to healthy longevity in Drosophila. Elife. (2018) 7:e35368. doi: 10.7554/eLife.35368

57. Miyo M, Yamamoto H, Konno M, Colvin H, Nishida N, Koseki J, et al. Tumour-suppressive function of SIRT4 in human colorectal cancer. $\mathrm{Br} \mathrm{J}$ Cancer. (2015) 113:492-9. doi: 10.1038/bjc.2015.226

58. Sun H, Huang D, Liu G, Jian F, Zhu J, Zhang L. SIRT4 acts as a tumor suppressor in gastric cancer by inhibiting cell proliferation, migration, and invasion. Onco Targets Ther. (2018) 11:3959-68. doi: 10.2147/OTT.S156143

59. Toska E, Osmanbeyoglu HU, Castel P, Chan C, Hendrickson RC, Elkabets $\mathrm{M}$, et al. PI3K pathway regulates ER-dependent transcription in breast cancer through the epigenetic regulator KMT2D. Science. (2017) 355:132430. doi: 10.1126/science.aah6893

60. Camarda R, Williams J, Goga A. In vivo reprogramming of cancer metabolism by MYC. Front Cell Dev Biol. (2017) 5:35. doi: 10.3389/fcell.2017.00035

61. Fisel P, Kruck S, Winter S, Bedke J, Hennenlotter J, Nies AT, et al. DNA methylation of the SLC16A3 promoter regulates expression of the human lactate transporter MCT4 in renal cancer 
with consequences for clinical outcome. Clin Cancer Res. (2013) 19:5170-81. doi: 10.1158/1078-0432.CCR-13-1180

62. Gatenby RA, Gillies RJ. Glycolysis in cancer: a potential target for therapy. Int J Biochem Cell Biol. (2007) 39:1358-66. doi: 10.1016/j.biocel.2007.03.021

63. Choi YK, Park KG. Targeting glutamine metabolism for cancer treatment. Biomol Ther. (2018) 26:19-28. doi: 10.4062/biomolther.2017.178

64. Cluntun AA, Lukey MJ, Cerione RA, Locasale JW. Glutamine metabolism in cancer: understanding the heterogeneity. Trends Cancer. (2017) 3:16980. doi: 10.1016/j.trecan.2017.01.005

65. Jiang J, Srivastava S, Zhang J. Starve cancer cells of glutamine: break the spell or make a hungry monster? Cancers. (2019) 11:E804. doi: 10.3390/cancers11060804

66. Medina MA, Nunez de Castro I. Glutaminolysis and glycolysis interactions in proliferant cells. Int $J$ Biochem. (1990) 22:6813. doi: 10.1016/0020-711X(90)90001-J

67. DeBerardinis RJ, Cheng T. Q's next: the diverse functions of glutamine in metabolism, cell biology and cancer. Oncogene. (2010) 29:313-24. doi: 10.1038/onc.2009.358

68. Kennedy SR, Zhang Y, Risques RA. Cancer-associated mutations but no cancer: insights into the early steps of carcinogenesis and implications for early cancer detection. Trends Cancer. (2019) 5:531-40. doi: 10.1016/j.trecan.2019.07.007

69. Yoshida K, Gowers KHC, Lee-Six H, Chandrasekharan DP, Coorens T, Maughan EF, et al. Tobacco smoking and somatic mutations in human bronchial epithelium. Nature. (2020) 578:266-72. doi: 10.1038/s41586-020-1961-1

70. Shen JC, Rideout WM, 3rd, Jones PA. The rate of hydrolytic deamination of 5-methylcytosine in double-stranded DNA. Nucleic Acids Res. (1994) 22:9726. doi: 10.1093/nar/22.6.972

71. Moore LD, Le T, Fan G. DNA methylation and its basic function. Neuropsychopharmacology. (2013) 38:23-38. doi: 10.1038/npp.2012.112

72. Piccolo FM, Fisher AG. Getting rid of DNA methylation. Trends Cell Biol. (2014) 24:136-43. doi: 10.1016/j.tcb.2013.09.001

73. He YF, Li BZ, Li Z, Liu P, Wang Y, Tang Q, et al. Tet-mediated formation of 5-carboxylcytosine and its excision by TDG in mammalian DNA. Science. (2011) 333:1303-7. doi: 10.1126/science.1210944

74. Evertts AG, Zee BM, Dimaggio PA, Gonzales-Cope M, Coller HA, Garcia BA. Quantitative dynamics of the link between cellular metabolism and histone acetylation. J Biol Chem. (2013) 288:12142-51. doi: 10.1074/jbc.M112.428318

75. Marmorstein R, Zhou MM. Writers and readers of histone acetylation: structure, mechanism, and inhibition. Cold Spring Harb Perspect Biol. (2014) 6:a018762. doi: 10.1101/cshperspect.a018762
76. Frye RA. Phylogenetic classification of prokaryotic and eukaryotic Sir2-like proteins. Biochem Biophys Res Commun. (2000) 273:7938. doi: 10.1006/bbrc. 2000.3000

77. Kori Y, Sidoli S, Yuan ZF, Lund PJ, Zhao X, Garcia BA. Proteomewide acetylation dynamics in human cells. Sci Rep. (2017) 7:10296. doi: 10.1038/s41598-017-09918-3

78. Hyun K, Jeon J, Park K, Kim J. Writing, erasing and reading histone lysine methylations. Exp Mol Med. (2017) 49:e324. doi: 10.1038/emm. 2017.11

79. D'Oto A, Tian QW, Davidoff AM, Yang J. Histone demethylases and their roles in cancer epigenetics. J Med Oncol Ther. (2016) 1:34-40.

80. Tian X, Zhang S, Liu HM, Zhang YB, Blair CA, Mercola D, et al. Histone lysine-specific methyltransferases and demethylases in carcinogenesis: new targets for cancer therapy and prevention. Curr Cancer Drug Targets. (2013) 13:558-79. doi: 10.2174/156800961131305 0007

81. Black JC, Whetstine JR. Tipping the lysine methylation balance in disease. Biopolymers. (2013) 99:127-35. doi: 10.1002/bip.22136

82. Zheng Y, Tipton JD, Thomas PM, Kelleher NL, Sweet SM. Site-specific human histone H3 methylation stability: fast K4me3 turnover. Proteomics. (2014) 14:2190-9. doi: 10.1002/pmic. 201400060

83. Monaghan L, Massett ME, Bunschoten RP, Hoose A, Pirvan PA, Liskamp RMJ, et al. The emerging role of $\mathrm{H} 3 \mathrm{~K} 9 \mathrm{me} 3$ as a potential therapeutic target in acute myeloid leukemia. Front Oncol. (2019) 9:705. doi: 10.3389/fonc.2019.00705

84. Zhao D, Zhang X, Guan H, Xiong X, Shi X, Deng H, et al. The BAH domain of BAHD1 is a histone H3K27me3 reader. Protein Cell. (2016) 7:222-6. doi: 10.1007/s13238-016-0243-z

85. Tsukada Y, Fang J, Erdjument-Bromage H, Warren ME, Borchers CH, Tempst $\mathrm{P}$, et al. Histone demethylation by a family of $\mathrm{JmjC}$ domain-containing proteins. Nature. (2006) 439:811-6. doi: 10.1038/nature04433

Conflict of Interest: The authors declare that the research was conducted in the absence of any commercial or financial relationships that could be construed as a potential conflict of interest.

Copyright (C) 2020 Ordway, Swietach, Gillies and Damaghi. This is an open-access article distributed under the terms of the Creative Commons Attribution License (CC $B Y)$. The use, distribution or reproduction in other forums is permitted, provided the original author(s) and the copyright owner(s) are credited and that the original publication in this journal is cited, in accordance with accepted academic practice. No use, distribution or reproduction is permitted which does not comply with these terms. 